\title{
DE LA SOCIEDAD INDUSTRIAL A LA SO CIEDAD DEL RIESGO (Una investigación sobre los tipos de crisis social en las sociedades complejas)
}

Josetxo Beriain

U niversidad Pública de $\mathrm{N}$ avarra

«La metáfora es la máscara de D ios mediante la cual puede experimentarse la eternidad.»

(J oseph Campbell, El poder del mito.)

«T enemos la impresión de estar ante dos tipos de realidades, ya en sí mismas distintas, y que una línea de demarcación netamente trazada las separa entre sí: por un lado, está el mundo de las cosas sagradas y, por el otro, el de las cosas profanas.»

(Emile D urkheim, Lasformas elementales de la vida religi osa.)

El objetivo fundamental de este trabajo es el análisis sociológico de «conceptos-límite» - crisis, riesgo, contingencia- , que establecen el nexo ${ }^{1}$ de observación, descripción e interpretación entre la sociedad instituida y la sociedad instituyente, es decir, el nexo que vincula selectivamente la experiencia social entre el espacio de experiencia del pasado y el horizonte de expectativas del futuro, articulando constelaciones de sentido socialmente plausibles. De esta guisa, voy a proceder a una tematización sociológica de estos conceptos, comenzando por el significado y función de las crisis.

1 N. LUHMANN, Beobachtungen der M oderne, O pladen, 1992, 99 ss.; Die W issenschaft der G esel schaft, Frankfurt/M , 1990, 68-122. 


\section{LA CRISIS SOCIAL-HISTORICA. CRISIS Y TIEMPO SOCIAL}

Voy a comenzar haciendo un breve recorrido de las significaciones históricas fundamentales del concepto de crisis, para proponer posteriormente un concepto de crisis que afecta a la sociedad. Tucídides muestra cómo, en la lucha de poderes, se trata de las batallas decisivas, de las cuales cuatro de ellas fueron decisivas para las guerras persas (el esquematismo operante es éxito/derrota). En la Escuela hipocrática se trata de la fase crítica de una enfermedad, en la que la lucha entre la vida o la muerte era definitivamente dirimida (el esquematismo operante es vida/muerte). A ristóteles muestra cómo, en el ámbito de la política, se trata de encontrar y defender el justo medio, donde participan todos los ciudadanos, pero también se trata de decisiones políticas, que todos deben presuponer como articuladas en torno al juicio justo (el esquematismo operante es juicio justo/juicio injusto). D entro de la Teología, sobre todo desde el Nuevo Testamento, la crisis, el Juicio ganan una nueva interpretación: el Juicio de Dios. La crisis significa «el Día del Juicio» al final de los tiempos (el esquematismo operante es salvación/condenación)². Voy a distinguir, siguiendo las investigaciones de Reinhart Kosselleck, cuatro modelos semánticos sobre la crisis3:

1. La historia puede ser interpretada como una crisis de duración. La historia mundial es la historia del Juicio del M undo. Se trata de un concepto de proceso. "La historia del mundo es el Juicio del Mundo», es una expresión de Schiller y al mismo tiempo el lema de una nueva Edad. Emerge en el pasaje de un verso de amor, en el que Schiller retoma una situación perdida que nunca más se podrá recuperar: «Lo que surge del minuto/no restituye ninguna eternidad»`. Formalmente se trata de la temporalización del día del Juicio Final, que siempre se realiza. La afirmación de Schiller tiene una gran pretensión: no sólo les es exigida una justicia inmanente a las historias particulares, sino a la totalidad de la historia mundial, in toto. Ya no existe el historiador que, ex post, bajo el fundamento de su mejor conocimiento dirige moralmente el pasado, sino la historia misma es situada como un sujeto actuante, que ejecuta la justicia. Así, Richard Rothe adapta, teleológicamente, finalísticamente la afirmación de Schiller cuando dice que: «Toda la historia cristiana en realidad es una gran crisis continuada del género humano»5; asimismo, $K$ arl Barth da un tono existencial a esta crisis de duración al afirmar: «La así Ilamada historia salvífica es sólo la crisis continua de toda historia, no una historia en o al lado de la historia» ${ }^{6}$.

2 R. Ko osselleck, «K rise», G eschichtliche G rundbegriffe, vol. 3, Stuttgart, 1982, 621-623.

3 R. KosSELLECK, «Einige Fragen an der Begriff von Krise», en R. Ko SSELLECK, Ü ber die Krise, Stuttgart, 1986, 68.

${ }^{4}$ H. SCHILleR, «Resignation. Eine Phantasie», Sämtliche W erke, E. V. der H ellen (comp.), Stuttgart, vol. 1, 199.

5 R. ROTHE, «Die Anfänge der christlichen Kirche und ihre Verfassung» (1837), citado por K OSSELLECK, Ü ber die Krise, 70.

${ }^{6}$ K. BARTH, Der Römerbrief, Zollikon-Zurich, 1954, 57, 32. 
2. La crisis puede señalar un proceso único y acelerador, en el que muchos conflictos afectan al sistema, para al final, después de la crisis, originar una nueva situación. La crisis es indicio del sobrepasamiento del umbral de una época, un proceso que puede repetirse «mutatis mutandis». Esta crisis hace referencia a un período iterativo. Cada pequeño o gran progreso posee su propia crisis, que actúa como generadora de progreso en la economía, en las ciencias naturales, en la técnica y en la industria.

3. Este concepto de crisis es, a diferencia de los otros, un concepto que hace referencia al futuro, y apunta a una decisión última. La consideración de «la crisis como decisión última» significa que la crisis de la que se trate hace referencia a la última, gran y única decisión, tras la cual la historia en el futuro aparecerá de forma totalmente diferente. Q uizás, trayendo a colación el ejemplo, sobre la evolución del capitalismo, de Karl M arx, resulte más comprensible este concepto de crisis. M arx, por una parte, esperaba con seguridad que la última crisis del capitalismo trajera consigo la liberación de la dominación y la extinción de las diferencias de clase; por otra parte, vio que las crisis del capitalismo, como sistema, no conducían en cuanto tales a una superación del sistema. El operaba, por una parte, con un concepto de crisis inmanente al sistema, en la cual se mostraba la estructura iterativa de las crisis económicas (sistémicas), y, por otra parte, operaba con una supuesta última lucha (elemento de decisión) entre el proletariado y la burguesía, que sin duda al guna, para él, sustituía al Juicio Final y evitaba, asimismo, una mera determinación económicosistémica.

4. Podemos entresacar un cuarto tipo de crisis $^{7}$, que se manifiesta en todas sus consecuencias en la modernidad tardía en la que estamos. Hoy día, «o inesperado socaba lo esperado». La tensión entre la experiencia y el horizonte de expectativas, que trae consigo nuevas resoluciones, se expande hasta conformar en el presente un «umbral de fragmentación»8 entre pasado y futuro. En la medida en que la doctrina cristiana del último Día sitúa un límite inamovible al horizonte de expectativas (hasta la mitad del siglo XVII), el futuro permaneció limitado al pasado. La revelación bíblica y la administración eclesiástica han limitado la tensión entre el espacio de experiencia y el horizonte de expectativas, de tal manera que se evitaba la ruptura de la continuidad entre ambos. Con la apertura de un nuevo horizonte de expectativas, el profectus espiritual fue desplazado o disuelto por un progresus mundano9. La doctrina del último Juicio es superada por el azar de un futuro abierto. La creciente asimetrización entre el espacio de experiencia y el horizonte de expectativas origina la crisis de la modernidad. El indicador incuestionable de que esta asimetría persiste es a través de su constante renovación, es decir, la aceleración ${ }^{10}$ de los ritmos temporales y de los intervalos da lugar a la crisis. El acortamiento cósmico

\footnotetext{
R. K OSSELLECK, Vergangene Zukunft, Frankfurt/M , 1979, 357 sS.

8 G. SIM M EL, D as Problem der H istorischen Zeit, Berlín, 1916, 29.

9 R. KoSSELLECK, op. cit., 362.

10 R. Ko osselleck, Ü ber die Krise, Stuttgart, 1986, 75.
} 
del tiempo, que antiguamente en lenguaje mítico debió preceder al Juicio Final, se deja verificar hoy empíricamente como aceleración de las secuencias históricas de los acontecimientos. En palabras de Jacob Burckhardt: «El proceso mundial conlleva una rapidez espantosa; los desarrollos que precisaban siglos se muestran en meses y semanas, como fantasmas volantes que pasan por delante, listos para pasar a la existencia» ${ }^{11}$. Podemos representar este concepto de «aceleración» a través de tres curvas temporales exponenciales:

1. a D esde hace cinco billones de años, el globo terráqueo tiene una corteza sólida; desde hace un billón de años existe vida orgánica en la tierra; desde hace diez millones de años existe vida humana, y desde hace dos millones de años se datan los primeros instrumentos fabricados.

2. a La segunda curva exponencial describe las primeras manifestaciones de arte genuino hace treinta mil años; el surgimiento de la agricultura y la ganadería data de hace diez mil años; de hace seis mil años data la cultura de las ciudades.

3. a En la tercera curva exponencial se incluye la sociedad industrial moderna desde hace trescientos años. La curva de aceleración se manifiesta a través de dos series de datos. Primero, la comunicación de la información se ha acelerado de tal manera que la identidad del acontecimiento y su noticia tienen una potencialidad de producción. Segundo, la aceleración de la circulación dentro del globo terráqueo unifica el tiempo y el espacio. Al mismo tiempo, esta curva exponencial multiplica la fuerza de autodestrucción. Con el incremento exponencial de posibilidades, también se produce un crecimiento exponencial de los riesgos, radicando aquí otra faceta de la crisis social, en la multiplicación de los riesgos ${ }^{12}$ : económicos, políticos, ecológicos, etc.

\section{CRISIS «REGIONALES»}

En la modernidad, «el presente se concibe como una transición hacia lo nuevo y vive en la conciencia de la aceleración de los acontecimientos históricos y en la esperanza de que el futuro será distinto $»^{13}$. La modernidad es la época que vive para el futuro, que se abre a sí misma a la novedad del futuro'it. Los nuevos procesos sociales tienen su propia estructura temporal; así se expresa H erder: «En la actualidad, toda cosa cambiante lleva consigo la medida de su propio tiempo... Existen en el universo, en cualquier momento, innumerables tiempos» ${ }^{15}$. Estos tiempos actúan así debido a la diferenciación funcio-

${ }^{11} \mathrm{~J}$. Burckhardt, Weltgeschichtliche Betrachtungen, R. Stadelman (comp.), Pfullingen, 1949, 211.

12 U. BECK, Ri sikogesel schaft, Frankfurt/M , 1986, 7-10.

$13 \mathrm{~J}$. H ABERM AS, Escritos políticos, Barcelona, 1988, 113.

14 J. H ABERM AS, D er Philosophische D iskurs der M oderne, Frankfurt/M , 1985, 15.

15 J. G. HERDER, M etakritik zur Kritik der Reinen Vernunft, Berlín, 1955, 68. 
nal ${ }^{16}$ de esferas sociales que caracteriza a las sociedades modernas. Ya no existe un lugar para una autorrepresentación holista de la sociedad. Esta no tiene una «cumbre» 0 un «centro»; más bien, está dividida en una multiplicidad de subsistemas interdependientes. Cada subsistema, así, la economía, la política, el sistema judicial, reconstruyen y «leen» su entorno en los términos de su propio lenguaje particular, tales como precios, poder, justicia. En este sentido, el origen de las crisis puede ser «regional», si afecta a alguno de los subsistemas sociales, y «global», si es la sociedad en su conjunto la que experimenta la crisis. Voy a analizar seguidamente las crisis que surgen en cuatro ámbitos sociales: la cultura, la economía, la política y la personalidad.

\subsection{La crisis de origen simbólico-cultural}

Si consideramos a la cultura como ese «proceso continuo de sustentación de una identidad mediante la coherencia lograda por un consistente punto de vida estético, una concepción moral del yo y un estilo de vida que exhibe esas concepciones en los objetos que adornan nuestro hogar y a nosotros mismos y en el gusto que expresan esos puntos de vista» ${ }^{17}$, tenemos que reconocer que la formación de esa identidad (social e individual) es selectiva y paradójica, ya que la reproducción de la identidad queda garantizada al nivel «taxonómico-clasificatori $0{ }^{18}$, es decir, al nivel de los bordes-fronteras que configuran las identidades económicas de las empresas, las identidades territoriales, las identidades políticas (los partidos), los roles, las posiciones, etc.; pero al nivel «arquetípico del sentido», al nivel de proporcionar identificaciones, a través de imágenes, metáforas, con el «Sí mismo» (Self, Selbst) queda interrumpido el proceso de reproducción de la identidad. En los términos de Bell, podíamos hablar de una «crisis espiritual». C omo ha señalado Clifford Geertz en La interpretación de las culturas (1973), se precisa una sintonización de las acciones humanas con un orden cósmico visualizado; en definitiva, unas formas simbólicas que articulen de una forma integrada la experiencia del hombre en el mundo.

Veíamos al comienzo de este trabajo cómo en las sociedades tribales el mito mediaba cognitivamente (simbólicamente, debiéramos decir) las contradicciones sociales que se daban entre la naturaleza y la cultura y dentro de ambas. Emile D urkheim descubría cómo dentro de la esfera de lo sagrado existían dos poderes o fuerzas ${ }^{19}$, unas «benéficas» y las otras «maléficas». Rudolf

${ }^{16} \mathrm{~N}$ iklas LUHMAN es quien con mayor éxito ha estudiado la dinámica de diferenciación en las sociedades modernas; así, en D ifferentiation of Society, N ew York, 1982, 230 ss.; Soziale Systeme, Frankfurt/M , 1984; 261 ss. T ambién se puede hallar una buena discusión del problema en P. FuCHS, Die Erreichbarkeit der G eselschaft, Frankfurt/M , 1992, 67-89.

17 D. BELL, Las contradicciones..., M adrid, 1977, 47.

${ }_{18}$ P. BouRdieu, Cosas dichas, Buenos Aires, 1988, 134 ss.

19 W. S. F. PICKERING, Durkheim's Sociology of Religion, London, 1984, 127-128; N. LUHMAN , Funktion der Religion, Frankfurt/M, 1977, 190. 
O tto mostró cómo la esfera máxima de trascendencia, el M isterio, Dios, se muestra como algo paradójico, ya que es algo tremendo y fascinante ${ }^{20}$. En ambos casos, un imaginario social central configura la identidad del grupo, proporcionando al individuo una «totalidad integrada de significado», a través de la cual el individuo encuentra las respuestas a preguntas fundamentales como son el significado de la muerte, de la moral, del terror, de lo bello, del sexo, del mal. En el judaísmo antiguo se racionaliza el problema del mal, como «mancha», «transgresión», «desvío», en definitiva, como «pecado», como «fisura real», a la que se contrapone la «gracia» 0 «sutura simbólica», en forma de esperanza de salvación. En la sociedad griega la tragedia es la protorrepresentación ritual colectiva, que asegura mágicamente la identidad colectiva de la existencia del clan frente al entorno. El H éroe representa la pasión y la muerte en que un hombre excedido por un destino debe sucumbir a su grandiosa función. En la estética clásica desde Aristóteles hasta $\mathrm{H}$ egel, la crisis designa el punto de inflexión de un proceso fatal, fijado por el destino, que, pese a su objetividad, no sobreviene simplemente desde fuera, ni permanece exterior a la identidad de las personas aprisionadas en él. La contradicción que se expresa en el apogeo catastrófico de un conflicto dramático es inherente a la estructura del sistema de acción y a los propios sistemas de personalidad de los héroes. El destino se cumple en la revelación de normas antagónicas, frente a las cuales sucumbe la identidad de los personajes cuando éstos se muestran impotentes para reconquistar su libertad doblegando el poder mítico del destino mediante la configuración de una nueva identidad. En la modernidad, el problema del mal aparece genialmente descrito en «la relación entre el amo y el esclavo» en la Fenomenología del espíritu de G. W. F. H egel, en la «alienación» descrita por $M$ arx, en la «angustia» descrita por K ierkegaard (y más tarde por D rewermann). En todos estos casos, el ser humano tiene que hacer frente a su «estado de arrojado al mundo» ${ }^{21}$, a lo característico de su condición humana. En todas las culturas, como afirma el mitólogo Joseph Campbell, la «ayuda», el «alivio» procede de un «Sí mismo» en el que se inscribe, con toda plausibilidad, la experiencia del hombre en el mundo. En el principio «todo era uno»»2, y después hubo una separación: cielo/tierra, bien/mal, macho/hembra, etc. Las culturas, en forma de mito y religión, han proporcionado la manera de mediación entre estas significaciones opuestas. U na vez que se han controlado las contingencias que afectan al sistema, todavía nos quedan las contingencias que afectan al ser humano, a su mundo de la vida. En clave religiosa, la esperanza de salvación, la negación de la contingencia existente entre el pecado y la gracia, depende de la relación entre ambas dualidades, mediadas por la decisión divina, y por la transformación de un elemento de la dualidad en el otro, por medio de una interacción social prescrita por el universo simbólico religioso, que actúa como reductor de contingencia.

\footnotetext{
20 R. O tTo, Lo santo, M adrid, 1985, 14 ss.

${ }^{21}$ M. H EIDEG GER, Sein und Zeit, Tübingen, 1986 (16), 179.

22 J. CAM PBELL, El poder del mito, Barcelona, 1991, 89, 93.
} 
La unidad (sagrada) de significado originaria (mana, wakan, orenda, etc.) se divide en otras unidades de significado de carácter autorreferencial, como son la ciencia, la moral, el derecho, el arte, etc., que se ubican en la esfera de lo profano. M ax Weber, en su Religionssoziologie, ha denominado a este proceso «racionalización de las cosmovisiones»; D aniel Bell lo ha denominado la «Gran Profanización», en el que se da una creciente autorreflexividad inherente a las nuevas representaciones colectivas autonomizadas de la esfera de lo sagrado. EI proceso de conformación de una «cosmovisión descentrada» ubicada en el ámbito de lo profano, nos pone de manifiesto una tendencia hacia la separación de los sistemas (en los términos de Erich N eumann), hacia el incremento de opciones, sin posibilitar una «vuelta al centro» (Sí mismo), desde el que se pueda equilibrar la separación de los sistemas. El «cocoon protector» de las grandes trascendencias (Th. Luckmann) se ha ocultado en formas no-simbólicas; éstas se han cristalizado en formas sin contenido. El signo (representación) sustituye al símbolo (interpretación) como portador de sentido. Actualmente, el discurso del miedo frente a las amenazas ecológicas, económicas, militares es un sustituto de las cosmovisiones holistas, en medio de la diferenciación funcional. El miedo no puede ser barrido por los sistemas políticos, económicos o militares; es auténtico e inmune a la refutación ${ }^{23}$. La «seguridad ontológica» del ser humano hace referencia a la confianza que la mayor parte de los seres humanos tenemos en la continuidad de nuestra identidad y en la continuidad de nuestros entornos sociales y naturales de acción ${ }^{24}$, es decir, el individuo tiene la experiencia del «Sí mismo» en relación a un mundo de personas y objetos organizados simbólicamente a través de la confianza básica.

\subsection{La crisis de reproducción económica}

En el capitalismo liberal, el vínculo funcional fundamental, anclado en el derecho privado, se da entre trabajo asalariado y capital. El núcleo institucional fundamental de tal capitalismo no es el Estado, sino el mecanismo de mercado: el intercambio económico es el medio de control dominante. La integración sistémica (en los términos de Parsons, la adaptación y la consecución de fines) se refiere al funcionamiento semiautónomo, de los mercados de trabajo, bienes y capital. Según el análisis de M arx, este modo de organización conduce a problemas estructuralmente insolubles, en forma de tendencias a la caída de la tasa de beneficios ${ }^{25}$ (crisis de acumulación de capital), y a una reducción de la capacidad de consumo y de los incentivos para invertir (crisis de realización de capital). Así, el ciclo de prosperidad, crisis y depresión es típico del capita-

${ }^{23} \mathrm{~N}$. LUHM AN N, Ö kologische Kommunikation, O pladen, 1986, 238.

${ }^{24}$ A. GIDDENS, The Consequences of M odernity, Stanford, 1990, 93; A. SCH ÜTZ, Las estructuras del mundo de la vida, Buenos Aires, 1977, 35-38.

${ }^{25}$ K. M ARX, El capital, M éxico, 1978, vol. 3, caps. XII, XIV y XV. 
lismo liberal. «L as ideologías burguesas pueden adoptar una estructura universalista y apelar a valores generalizables porque el régimen de propiedad se ha despojado de la forma política y se ha traspasado a una relación de producción que... puede legitimarse a sí misma: la institución del mercado puede apoyarse en la justicia inherente al intercambio de equivalentes. Por eso, el Estado constitucional burgués... tiene su justificación en las relaciones de producción legítimas, éstas pueden prescindir de un poder tradicional legitimado "desde lo alto" $\rangle^{26}$. Las luchas del proletariado tienen un carácter «reactivo», de resistencia frente al nuevo orden capital ista que desmantela los lazos artesanales y comunitarios ${ }^{27}$; eran luchas al margen de y contra la embestida del capital y el Estado central.

La legitimación ya no viene primariamente «de arriba» (de las cosmovisiones tradicionales), sino «de abajo» (de la «justicia» inmanente al mercado). La relación de clase queda institucionalizada a través del mercado de trabajo, y, por tanto, «despolitizada». «Puesto que la coacción social ejercida por los capitalistas se ha institucionalizado en el contrato de trabajo privado como relación de intercambio, y la extracción de plusvalía sobre la que disponen los particulares ha reemplazado a la dependencia política, el mercado, además de su función cibernética, tiene una función ideológica: la relación de clases se vuelve anónima en la forma no política de su dependencia salarial $\gg x^{8}$.

En el capitalismo tardío la relación entre el Estado y la economía ha cambiado. $\mathrm{H}$ an surgido nuevos fenómenos sociales: los créditos gubernamentales, la redistribución de ingresos, las subvenciones, los préstamos, las contratas, los precios garantizados, la política laboral por medio de la cual se hacen los ajustes entre imperativos antagónicos que representan el crecimiento sostenido, la estabilidad de la moneda, el pleno empleo y el equilibrio del comercio exterior, la organización gubernamental de bloques económicos supranacionales, el consumo improductivo (en armamento y programas espaciales, por ejemplo), la mejora de las infraestructuras materiales e inmateriales (transporte, comunicación, vivienda, $I+D$ ), la mejora de la productividad del trabajo (escuelas profesionales, programas de capacitación) y la compensación de los costes sociales de la producción privada (seguro de desempleo, salario social, atención al equilibrio ecológico), a través de los cuales se abren y mejoran las oportunidades de inversión de capital y se aumenta la productividad del trabajo ${ }^{29}$. Estos fenómenos producen dos cambios fundamentales: 1) la actividad gubernamental ha alterado la forma de producción de valor excedente; al tener que remediar las fallas funcionales del mercado, el Estado interviene en el proceso de acumulación de capital, y 2 ) en amplios sectores de la economía el mecanismo de mercado ha sido sustituido por un «compromiso cuasipolítico» entre los empresa-

26 J. H ABERM AS, L os problemas..., 1975, 39.

27 J. O'CONnOR, Crisis de acumulación, Barcelona, 1987, 51,60,64; Ch. TILLY, From M obilization, N ew York, 1978, 145-146.

28 J. H ABERM AS, op. cit., 43.

29 Th. M CCARThy, La teoría crítica de ürgen H abermas, M adrid, 1987, 420. 
rios y los sindicatos en lo concerniente a la determinación de los costes de la fuerza de trabajo, pero este compromiso es precario por la atomización de intereses que se deriva de la ruptura de las identidades de clase, como han puesto de manifiesto J. Elster, en El cemento de la sociedad (1989), y M. O Ison, en The Logic of Collective Action (1965). No obstante, el reequilibrio entre Estado y economía que produce la «sociedad de suma positiva» ${ }^{0}$ es ficticio, ya que, más que al «Estado de Bienestar», tenemos que hacer frente a más «E stado de Bienestar»; en definitiva, el aparato del Estado no puede, bajo condiciones marginales dadas, controlar adecuadamente al sistema económico, produciéndose así una crisis de «output». Por otra parte, la utopía de la «sociedad centrada en el trabajo» ya ha perdido su poder de convicción, no sólo porque las fuerzas productivas hayan perdido su inocencia, o porque la abolición de la propiedad privada no desemboque por sí misma en la autogestión obrera; la utopía productivista se ha ocultado por la razón de que la fuerza determinante de realidades como el trabajo, la producción y el mercado es cada vez menos visible en relación con la constitución de la sociedad en su conjunto. La crisis de la sociedad del trabajo se pone de manifiesto en la propia diferenciación interna del trabajo asalariado, en el declive de la ética del trabajo y en la emergencia de una sociedad postclasista individual ista de productores ${ }^{31}$.

El Estado de Bienestar keynesiano es una víctima de su propio éxito. Eliminando y suavizando las crisis, ha inhibido la función positiva que las crisis realizaban en el proceso capitalista de «destrucción creativa» ${ }^{32}$. Comporta la consecuencia no deseada pero innegable de socavar ambos incentivos, a la inversión y al trabajo. Podemos concluir así: las crisis económicas están latentes en el capitalismo ${ }^{33}$.

\subsection{La crisis de legitimación}

La necesidad de distribuir el producto social de forma desigual $y$, sin embargo, legítima obliga al Estado a una «repolitización de las relaciones de producción»; ya no basta con apelar a la «justicia» inmanente del mercado, es necesario recurrir a algún "programa sustitutivo»; así, el Estado subvenciona y reemplaza al mercado allí donde éste fracasa en sus funciones de producción y acumulación. Este nuevo rol del Estado genera la consecuencia no pretendida de sobrecargarlo con expectativas que no pueden ser realizadas, incrementándose así la demanda de justificación pública del Estado a los ojos de los ciudadanos, ya que no genera las adhesiones suficientes, en forma de lealtad de

${ }^{30} \mathrm{Cl}$. O FFE, «The Challenge of the N ew Social M ovements», en Social Research, 1985, 52, 4, 822; A. W o LFE, Whose Keeper?, Berkeley, 1989, 151 ss.

${ }_{31} \mathrm{Cl}$. Offe, D isorganized Capitalism, Cambridge, M ass., 1985, 134 ss.; U. BECK, Risikogeselschaft, Frankfurt/M , 1986, 205-220; Cl. O FFE, La sociedad del trabajo, M adrid, 1992.

$32 \mathrm{Cl}$. O FFE, Contradictions of the W elfare State, C ambridge, M ass., 1984, 199.

${ }^{33} \mathrm{~J}$. O'CONNOR, The M eaning of C risis, London, 1987, 68. 
masas, teniendo que hacer frente a una crisis de input. El consenso social que caracterizaba a la «sociedad de suma positiva» deviene así un «cuello de bote$\| a »^{34}$, ya que el «nuevo interventor» recibe presiones de todos los actores sociales. Por otra parte, las instituciones y procedimientos de la «democracia formal» aseguran un sentimiento de adhesión difuso y generalizado de la población. El espacio público, cuyas funciones han quedado reducidas en buena parte a plebiscitos periódicos en los que puede concederse o negarse la aclamación, está «estructuralmente despolitizado»»" La pacificación del mundo del trabajo, a través de una normalización del rol de trabajador y de una reevaluación del rol de consumidor, tiene su correlato en el equilibrio que se establece entre el rol de ciudadano, generalizado a la vez que neutralizado, y el inflado rol de cliente de las burocracias.

\subsection{El repliegueindividualista al yo}

Esta despolitización tiene como contraparte una reacción que se manifiesta como una tendencia hacia el «privatismo cívico» ${ }^{36}$ que consiste en la abstención política combinada con una orientación en función de la carrera, del ocio y del consumo que fomenta las expectativas de recompensa conformes con el sistema (ingresos, tiempo libre y seguridad); el correlato de este privatismo cívico es un privatismo familiar profesional que consiste en una orientación en función de la familia, caracterizada por intereses de consumo y ocio conspicuos ${ }^{37}$, por un lado, y en una orientación en función de la carrera profesional adaptada a la competencia por conseguir buenos puestos, por el otro. Este proceso de «privatización» ${ }^{38}$ ha sido estudiado como un proceso social generalizado por Richard Sennett ${ }^{39}$, por Christopher Lash ${ }^{40}$ y por H elena Béjar, El ámbito de lo íntimo (1988). Cada persona, retirada dentro de sí misma, se comporta como si fuese un extraño al destino de todos los demás. Sus hijos y sus buenos amigos constituyen para él la totalidad de la especie humana. En cuanto a sus relaciones con sus conciudadanos, puede mezclarse entre ellos, pero no los ve; los toca, pero no los siente. El existe solamente en sí mismo y para él solo. Y si en estos términos queda en su mente algún sentido de familia, ya no persiste ningún sentido de sociedad ${ }^{41}$.

${ }^{34}$ Cl. O fFE, Contradictions..., 1984, 72; J. ELSTER, La sociedad de cemento, Barcelona, 1991, 68-119.

35 J. H ABERM AS, T eoría de la acción comunicativa, M adrid, 1987, vol. 2, 494.

36 J. H ABERM AS, L os problemas..., 1975, 96.

37 Como han analizado Th. Veblen, The Theory of Leisure Class, New York, 1979, y P. BOURDIEU, La distinción, M adrid, 1988.

${ }^{38} \mathrm{Q}$ ue ha sido descrito en análisis dedicados a la religión por Th. LUCKMANN, Die Unsichtbare Religion, Frankfurt/M , 1991, y por N . LuH M AN N, Funktion der Religion, Frankfurt/M , 1977.

39 R. Sennett, Narcisismo y cultura moderna, Barcelona, 1980, 37-105.

${ }^{40} \mathrm{Ch}$. LASH, The Culture of N arcisism, N ew York, 1979.

${ }^{41}$ A. de Tocqueville, citado en R. SEn N ETT, El declive del hombre público, Barcelona, 1978, 6. 
Sennett describe un proceso que estructura la personalidad y que comienza a manifestarse en la sociedad victoriana y llega hasta hoy día, y que conduce a una «Gemeinschaft destructiva», a una sobrevaloración represiva de la esfera íntima. La privacidad se transforma en intimidad ${ }^{42}$.

La privatización de la experiencia familiar en el siglo XIX significa, por un lado, la creencia de que habría que trasladar a la familia fuera de las agitaciones del mundo externo y constituir una esfera moral más elevada y, por otro lado, significa un código de interacción humana derivado de la creencia en la personalidad inmanente que vuelve a arrojar a la familia en las mismas ansiedades sobre el orden que regían la vida pública. La familia, como esfera íntima, hoy día se ha fragmentado al incorporarse la mujer al mercado de trabajo y al flexibilizarse las rupturas matrimoniales (divorcios) y sentimentales, creando nuevas situaciones para los hijos, al tener que vivir con el padre o con la madre, complicándose la situación con nuevas nupcias, en donde los hijos del matrimonio anterior conviven con los hijos del matrimonio actual configurando un mapa de relaciones familiares de intimidad precaria.

El mundo social aparece como un «espejo del yo» ${ }^{43}$, en donde éste se refleja narcisísticamente a sí mismo, hasta el infinito. El yo de la modernidad tardía es un «yo proteico» ${ }^{44}$ que es totalmente inmanente al mundo y se apoya en las apariencias y sensaciones inmediatas, en la simultaneidad y en el impacto. De aquí el interés por el cuidado del cuerpo, de la piel, del look, como envolturas de un yo que se refleja a sí mismo, en una sociedad que como «otro generalizado» se ha ocultado proyectando una imagen de «vacío», «nada», de «abismo», donde predomina una mera trascendencia especular (speculum). Un «yo proteico» puede llevar una vida inmediata rica, pero sólo a costa de su acomodo al medio que le rodea. Este «repliegue narcisista» al «yo privado-íntimo» es una huida del «yo público», que ha sido incluido $0^{45}$ en los sistemas funcionales como fuerza de trabajo, como consumidores, como obligados a pagar impuestos y como asegurados, como electores, como individuos en edad escolar... La inclusión progresiva en cada vez más sistemas funcionales no significa un aumento de autonomía; este tipo de individualización social aísla o singulariza, pero no individúa en sentido enfático ${ }^{46}$. Como afirma Ch. Lash, «political and economic solutions don't work»; por tanto, «vivir el momento» ${ }^{47}$ es la pasión predominante, vivir para uno mismo, no para los predecesores, ni para los sucesores.

\footnotetext{
42 R. Sennett, Narcisismo..., Barcelona, 1980, 76 SS.

${ }^{43}$ R. SenNett, op. cit., 51.

${ }^{44}$ R. Sennett, op. cit., $59 \mathrm{~S}$.

${ }^{45}$ N . LuH m AN N, Politi sche Theorie im W ohlfahrtstaat, M ünchen, 1981, 25 ss.

46 U. BECK, Risikogeselschaft, Frankfurt/M , 1986, 207.

${ }^{47} \mathrm{Ch}$. LASH, The Culture of $\mathrm{N}$ arcisism, N ew York, 1979, 30.
} 


\section{CRISIS «GLOBALES»}

Una vez que he analizado el surgimiento de las crisis en diferentes sistemas sociales de acuerdo a principios inherentes a los propios sistemas, voy a examinar a continuación el surgimiento de la crisis como un «hecho social total» ${ }^{48}$, que se produce como la disyunción de principios de organización, como la colonización del mundo de la vida por el sistema, y como la contraposición entre dos modernizaciones, la de la expansión de opciones y la de la expansión de los riesgos.

\subsection{La crisis como disyunción de principios de organización social}

En la evolución de las sociedades, la diferenciación funcional de sistemas social es ha originado una dinámica nueva al producir nuevas realidades institucionales - la empresa capitalista, el Estado moderno, la cultura de masas- , a su vez diferenciadas ellas mismas. Así, por ejemplo, aparece la cultura moderna con sus representaciones colectivas plurales frente a la conciencia colectiva tradicional de carácter homogéneo, como han analizado Durkheim y Weber; la economía se ha diferenciado, asimismo, entre trabajo asalariado y capital, como $\mathrm{M}$ arx lo ha descrito, y el Estado se diferencia, también, en parte política y en parte administrativa. Parsons nos ha proporcionado una tabulación cruzada de los intercambios entre estos subsistemas, a través de medios simbólicos generalizados de comunicación, y Luhmann nos proporciona una teoría general de la diferenciación social, que afecta a todos los subsistemas sociales y sus entornos. No obstante, la diferenciación conduce a problemas insolubles en la coordinación de los subsistemas. Así, D aniel Bell afirma que «las contradicciones del capitalismo se manifiestan en los principios antagóni cos que subyacen a las estructuras tecnoeconómica, política y cultural... La esfera económica se basa en el principio axial de economizar: el esfuerzo para conseguir eficiencia a través de la organización de las actividades en pequeños componentes de coste unidad-beneficio, tal como se define en los sistemas de contabilidad financiera. La estructura axial basada en la especialización y la jerarquía es la coordinación burocrática. N ecesariamente, los individuos no son tratados como personas, sino como "cosas" (sus conductas están reguladas por requerimientos de rol), como instrumentos para maximizar beneficios. Los individuos se disuelven en sus funciones... La esfera política que regula el conflicto es gobernada por el principio axial de la igualdad: igualdad ante la ley, igualdad de derechos civiles y, más recientemente, igualdad de derechos económicos y sociales. Debido a que estas pretensiones se traducen en posibilidades legales de intervención, el orden político interviene en las esferas económicas y sociales (en los asuntos de las corporaciones, de las universidades y de los hospitales), en orden a recon-

${ }^{48}$ M. M Auss, Sociología y antropología, M adrid, 1978, 157. 
ducir las posiciones y las recompensas generadas en la sociedad por el sistema económico. La estructura axial de la política es la representación, y más tarde la participación. Las tensiones entre igualdad y burocracia marcan los conflictos de hoy... Finalmente, la esfera cultural es aquella de la autoexpresión y de la autogratificación. Es antiinstitucional y antinómica, por la razón de que el individuo es situado como la medida de satisfacción y sus sentimientos y juicios son los que determinan el valor de los objetos culturales. En esta democratización de la cultura, todo individuo busca realizar su potencial completo y, de esta manera, el "sí mismo" individual entra en conflicto con los requerimientos de rol del sistema tecnoeconómico ${ }^{49}$. Parece como si la modernización de estos subsistemas se realizase a costa de la modernidad del todo. Precisamente debido a la «apertura al futuro» de los subsistemas y de las innovaciones intensivas de sus racionalidades sectoriales, la sociedad misma parece incapaz de concebir su propio futuro como un proyecto. Claus $\mathrm{O}$ ffe plantea un análisis de la crisis en un artículo de $1971^{50}$, cuando describe en el capitalismo tardío una disyunción organizacional entre el sistema político-administrativo, el sistema económico y el sistema normativo, considerando que el código de intercambio input-output muestra una «incapacidad sistémica» (déficit) del sistema político-administrativo para coordinar las acciones de los tres subsistemas. En un artículo posterior, de 198651, O ffe completa su diagnóstico considerando que la capacidad de autogobierno que afecta a los sistemas de las sociedades modernas se vuelve problemática cuando coinciden tres factores: 1) El factor de una creciente diferenciación incrementa, a su vez, la vulnerabilidad y dependencia de cada ámbito social en relación a otros ámbitos y produce así la necesidad de una coordinación global. 2) La falta de adecuación de los medios y mecanismos de coordinación disponibles. 3) La inflexibilidad y la resistencia a la revisión que los mecanismos disponibles de coordinación sitúan frente a todo intento de cerrar la fisura entre las necesidades de autogobierno en los sistemas y las capacidades de dichos sistemas, entre el «diseño de la complejidad» y el «control de la complejidad» a través de innovaciones institucionales.

3.2. La crisis como expresión de la colonización del mundo de la vida por el sistema

Este enfoque teórico para delimitar la crisis social lo encontramos en dos obras de Jürgen $\mathrm{H}$ abermas, Los problemas de legitimación en el capitalismo tardío (1973) y en la Teoría de la acción comunicativa (1981). H abermas delimita un concepto de sociedad en dos niveles: sistema y mundo de la vida, ofrecien-

49 D. BELL, «M odernism and Capitalism», Partisan Review, 1978, 4, 211-212; Las contradicciones..., M adrid, 1977, 23 ss.

${ }^{50}$ Artículo recogido en Cl. O fFE, Contradictions..., Cambridge, M ass., 1984, 52.

${ }^{51} \mathrm{Cl}$. O FFE, «D ie U ttopie der N ull O ption», Soziale W elt, 1986, 4, 105. 
do una conexión entre ambos conceptos de sociedad. La integración sistémica hace referencia a los modos de coordinación de la acción social a través de la interconexión funcional de consecuencias de acción y puede darse aun cuando haya di screpancias entre las intenciones y consecuencias; la integración social se refiere a la coordinación de la acción a través de la comprensión - comunicativamente mediada - de orientaciones de acción y no puede tener lugar a menos que las consecuencias de la acción sean compatibles con las intenciones de los actores sociales ${ }^{52}$. Los sistemas de acción sólo pueden ser considerados desde la perspectiva externa de un tercero, del observador; Ia integración social debe ser analizada desde la perspectiva interna de aquellos envueltos, de ego y de alter ${ }^{33}$. Ambas formas de integración son senderos complementarios de racionalización. Existen un rango de constelaciones en las que sistema y mundo de la vida pueden ser interrelacionados entre sí: «0 las instituciones a través de las cuales los mecanismos de autogobierno como el dinero y el poder, estando anclados en el mundo de la vida, canalizan la influencia que el mundo de la vida ejerce sobre los sistemas de acción formalmente organizados, o tales instituciones canalizan la influencia que los sistemas ejercen sobre el mundo de la vida. En el primer caso, las instituciones funcionan como el marco que sujeta el mantenimiento del "sistema" a las "restricciones" normativas del mundo de la vida; en el segundo caso, ellas funcionan como la base que sujeta el mundo de la vida a las constricciones de la reproducción material, y, por tanto, lo mediatizan $»^{54}$. El origen de la crisis no radica en el «efecto desestructurador» del desencantamiento del mundo (Weber), ni en el proceso de diferenciación funcional de sistemas sociales (D urkheim), ni en el efecto de alienación económica por la equiparación de las relaciones sociales a relaciones entre cosas (M arx, Lukács), sino que, más bien, el origen de la crisis debe buscarse en la penetración de formas de racionalidad económica y administrativa en ámbitos del mundo de la vida55. Los principales puntos de referencia para $\mathrm{H}$ abermas son $M$ arx y Weber. Los fenómenos a los que había aludido Weber con su visión de la iron cage, y que los marxistas han descrito en términos de «reificación», son situados ahora en las relaciones selectivas de intercambio que se dan entre sistema y mundo de la vida, a través de la inclusión de los individuos en los roles de trabajador, consumidor, ciudadano y cliente de las burocracias. A través de estos canales y sirviéndose de medios de comunicación simbólica generalizada - dinero, poder - , el mundo de la vida es subordinado a imperativos sistémicos, la vida diaria es crecientemente monetarizada y burocratizada ${ }^{56}$. A esta específica mediatización sistémica es a lo que H abermas llama «colonización del mundo de la vida por los sistemas» abstractos que operan como sustitutos altamente especializados del lenguaje ordinario. Esta es una «interac-

\footnotetext{
52 J. H ABerm AS, L os problemas..., 1975, 17-18; Cl. O FFE, Contradictions..., 1984, 81.

53 J. H ABERM AS, T eoría..., M adrid, 1987, vol. 2, 26 ss.

54 J. H ABERM AS, op. cit., 261-262.

55 J. H ABERM AS, op. cit., 469.

$56 \mathrm{~J}$. H ABERM AS, op. cit., 453 ss., 474, 494.
} 
ción sin mundo», donde los sujetos estratégicamente ejercen una influencia externa entre sí. El «sistema» interviene en el «mundo de la vida», por una parte, monetarizando la conducta de las personas en la forma de «lógica mercantil», el dinero convierte el «intercambio simbólico» entre las personas en compra-venta, al actuar como un «sustituto técnico de Dios» ${ }^{57}, y$, por otra parte, burocratizando las conductas humanas a través del poder convirtiendo al sujeto en objeto de disciplina, de control y de vigilancia ${ }^{58}$. Los medios abstractos - dinero y poder - ponen entre paréntesis el tiempo y el espacio, así como a las personas, desplegando modos de conocimiento técnico que tienen validez independientemente de los que los usan ${ }^{59}$.

\subsection{La crisis como «soci edad del riesgo»}

Cuando hablamos de sociedad industrial (avanzada), pensamos frecuentemente en una realización institucional de la modernidad, pero, en realidad, tal sociedad representa una modernidad, la de la producción selectiva de riqueza y opciones; la otra modernidad es la del riesgo ${ }^{60}$; la sociedad del riesgo es la época del industrialismo en la que los hombres han de enfrentarse al desafío que plantea el conglomerado industrial para destruir, directa o indirectamente, toda forma de vida sobre la tierra y su dependencia de ciertas decisiones ${ }^{61}$. Este siglo es muy rico en catástrofes históricas (no naturales); así, dos guerras mundiales, Auschwitz, N agasaki, H arrisburg, Chernobyl, etc. Todo sufrimiento, toda miseria, toda prueba de fuerza que los seres humanos han ocasionado estaban relacionados con los «otros» - obreros, judíos, negros, refugiados, disidentes, mujeres, comunistas, etc.- , lo cual dejaba a salvo, al menos en apariencia, al resto. Ahora, sin embargo, nos encontramos ante «la desaparición de los otros ${ }^{62}$, ante la desaparición de nuestras muy preciadas posibilidades de distanciamiento: la distancia se ha esfumado ante la contaminación atómica y química y ante una expansión de la contingencia en ámbitos políticos, económicos y culturales. La miseria puede ser marginada, pero los peligros que se derivan de la era atómica y química no. En esto consiste la «omnipotencia del peligro». Lo más íntimo - por ejemplo, el cuidado de un niño- y lo más distante, generalizado - un accidente nuclear en un reactor en U crania- , están,

${ }^{57}$ K. BuRKe, A Grammar of M otives, Berkeley, 1969, 108-113.

58 M. FOUCAULT, Vigilar y castigar, M adrid, 1977, 142.

59 A. GIDDENS, M odernity and Self-I dentity, London, 1991, 18.

60 Esta idea ha sido desarrollada de forma sistemática por U. BECK, Risikogeselschaft, Frankfurt/M , 1986; G egengifte, Frankfurt/M , 1989; Politik in der Risikogeselschaft, Frankfurt/M , 1991; «La irresponsabilidad organizada», en D ebats, marzo-junio 1991, 31-37; «From Industrial Society to Risk Society», Theory, C ulture and Society, vol. 9, 1992, 97-123.

${ }_{61}$ U. BeCK, «La irresponsabilidad organizada», Debats, 1991, 31; N. Luhmann, Soziologie des Risikos, O pladen, 1991.

62 U. BECK, op. cit., 31; Risikogesel schaft, 1986, 7. 
ahora, de repente, conectados ${ }^{63}$. El peligro nos convierte a todos en vecinos de Chernobyl, en ciudadanos de la CEl.

En las sociedades desarrolladas, las restricciones sociales derivadas del nacimiento son eliminadas a través de la propia decisión y del rendimiento dentro de una ocupación-función social, pero con la producción social ${ }^{64}$ de estos nuevos riesgos surge un nuevo destino peligroso de carácter «adscriptivo», que no es ninguna reliquia del pasado, como en principio pudiera parecer, y que nos sitúa, paradójicamente, ante una Edad M edia moderna del peligro. Parafraseando a Adorno y H orkheimer en la D ialéctica de la Ilustración (1944), podemos decir que «el riesgo, como secularización de la fortuna de las soci edades tradicionales, revierte en mitología», ya que su calculabilidad es indeterminada. Aaron Wildavsky, en Searching for Safety $(1988)^{65}$, describe dos estrategias universales para obtener seguridad que operan ampliamente en áreas muy variadas, como la vida no humana, el cuerpo humano, el poder nuclear y la regulación jurídica de agravios. La primera estrategia es la «capacidad adaptativa» (resilience) y la segunda la «anticipación». La «capacidad adaptativa» opera de acuerdo con el principio de ensayo y error: un sistema actúa primero, corrige los errores cuando aparecen y, así, acumula seguridad a través del aprendizaje al hacerlo. La «anticipación» opera de forma opuesta: un sistema intenta evitar previamente las amenazas situadas como hipótesis, y no permite ensayos sin garantías previas contra el error. La posición de W ildavsky se puede resumir: «N o safety without risk». La simple constatación de que las causas del riesgo y la seguridad no son independientes, sino interdependientes, proporciona una enérgica herramienta para mostrar que un énfasis desmedido sobre la seguridad anticipatoria pudiera generar nuevos riesgos y precipitadamente impedir «beneficios de oportunidad» potenciales procedentes de las nuevas tecnologías, mientras que el asumir riesgos puede desarrollar la seguridad a través de la acumulación de conocimiento y de recursos. Esta tesis de afrontar riesgos a través de la capacidad adaptativa no hace sino confirmar la indeterminación de la calculabilidad del riesgo. Niklas Luhmann, en su texto Ö kologische Kommunikation (1986) ${ }^{66}$, apunta la tesis de que la sociedad moderna, debido a su diferenciación estructural, genera no suficiente y demasiada resonancia sobre los riesgos ecológi $\cos ^{67}$. La sociedad moderna no posibilita una representación holista de la sociedad; por tanto, las amenazas ecológicas son tematizadas y fragmentadas por los subsistemas funcionales de acuerdo a sus códigos binarios específicos - «verdadero versus falso» en la ciencia, «gobierno versus oposición» en la política, «posesión versus no posesión» en la economía一 , en lugar de ser abor-

${ }^{63} \mathrm{U}$. BECK, «The Anthropological Shock: Chernobyl and the contours of Risk Society», Berkeley Journal of Sociology, 32, 1987.

64 M. Douglas y A. W ILdavsky, Risk and Culture, Berkeley, 1982, 16-49, 186 ss.;

S. KRIM SKY y D. GOLDIN G, Social Theories of Risk, W esport, Conn., 1992, 83-117.

${ }^{65}$ A. W ILD AVSKY, Searching for Safety, N ew Brunswick, 1988.

${ }^{66}$ N . LUHM AN N, Ö kologische Kommunikation, O pladen, 1986.

${ }^{67}$ N. LUHMAN N, op. cit., 220. 
dados por la sociedad como un todo: la sociedad no genera bastante resonancia de los riesgos ecológicos. Al mismo tiempo, estos riesgos globales tienden a sobrecargar las capacidades para resolver problemas de cada subsistema. D ebido a que la diferenciación funcional implica una pérdida de redundancia entre los subsistemas, pudiera ocasionar reacciones en cadena incontroladas en los otros subsistemas: la sociedad moderna genera demasiada resonancia a los riesgos ecológicos. El discurso sobre el miedo ${ }^{68}$ de los nuevos movimientos sociales, de Los Verdes y de gran parte de la sociedad civil es un sustituto de las cosmovisiones holistas. El miedo se proyecta como la reacción de un «mundo sin hogar» frente a la contingencia, es decir, frente a la negación de la necesidad y de la imposibilidad del riesgo como construcción social, o frente a la «probabilidad de lo improbable». En otro contexto, el de la triple transición en Europa Central y $O$ riental, se pone de manifiesto la contingencia total que se deriva de la confluencia simultánea de tres procesos: la construcción de la casa común (la cuestión de la identidad), la glasnost (la cuestión de la democracia) y la perestroika (la cuestión de la organización económica), ante los que no existe un «diseño de complejidad» que determine quiénes son los actores, qué tipo de acciones, en qué circunstancias y con qué objetivos puede funcionar el nuevo orden postrevolucionario ${ }^{69}$.

\section{A MODO DE CONCLUSION}

Después de efectuar el recorrido sobre los diferentes tipos de crisis, podemos entresacar algunas notas finales:

1. Las contradicciones sociales son inherentes a los propios procesos de estructuración de la sociedad; así, hemos distinguido entre contradicciones existenciales y contradicciones estructurales.

2. Las crisis son igualmente inherentes a los procesos de estructuración de la sociedad, pero sólo son percibidas como tales cuando afectan a la experiencia real de las personas, cuando ponen en peligro el mundo de la vida dado por supuesto; no importa que tengan un origen «regional» 0 que sean un «hecho social total».

3. La temporalización ${ }^{70}$ de las crisis hace que surjan «umbrales de fragmentación» y «umbrales de conjunción» de opciones, posibilidades y riesgos. La mayor o menor continuidad entre el antes y el después son su condición de posibilidad.

68 N. Luhmann, op. cit., 237-249; E. Drewermann, Strukturen des Bösen, Padernborn, 1988, vol. 3, 438 .

${ }_{69} \mathrm{Cl}$. O FFE, «C apitalism by D emocratic D esign?», Social Research, vol. 58, 4, 1991, 866.

70 N . LuH M AN N, «Risiko und G efahr», Soziologische Aufklärung, vol. 5, O pladen, 1990, 158159. 
4. Así como en el siglo XIx la modernización ha creado la imagen estructural de la sociedad industrial, disolviendo la sociedad estamental agraria, la modernización disuelve los contornos de la sociedad industrial y la continuidad de la modernidad origina otra configuración social.

\title{
RESU MEN
}

Este trabajo hace referencia al concepto sociológico de crisis, distinguiendo entre dos tipos principales de crisis: el «regional» y el «global». Esto significa que tenemos que considerar un concepto de sociedad en dos niveles: por una parte, la sociedad funcionalmente diferenciada en subsistemas, y por otra parte, la concepción holista de la sociedad como un todo, para poder describir los posibles tipos de crisis social. Las crisis en la sociedad moderna surgen bajo los constructos de sobrecomplejidad, contingencia y riesgo.

\begin{abstract}
ABST RAC T
This papper focuses on the key sociological concept of crisis, differentiating two main types of crisis: the «regional» one and the «global» one. That means that we have to take into account a two-levels concept of society, on the one hand, the functionally differentiated society in subsystems, and on the other hand, the holistic conception of society as a whole in order to get an account of the posible types of social crisis. The crisis in modern societies arises under the constructs of overcomplexity, contingency and risk.
\end{abstract}


TEXTO CLASICO 